\title{
The fermion-fermion effective potential in the Maxwell-Chern-Simons theory it
}

\author{
H.O. Girotti \\ Instituto de Física, Universidade Federal do Rio Grande do Sul, C.P. 15051, 91500 Pôrto Alegre RS, Brazil
}

\author{
M. Gomes and A.J. da Silva \\ Instituto de Física, Universidade de São Paulo, C.P. 20516, 01498 São Paulo SP, Brazil
}

Received 18 September 1991

The effective nonrelativistic potential $V_{\mathrm{T}}$ describing the fermion-fermion interaction in the Maxwell-Chern-Simons theory is derived to the lowest order in perturbation theory. As expected, $V_{\mathrm{T}}$ is not invariant under parity and time-reversal transformations. The quantum dynamics generated by $V_{T}$ becomes exactly solvable at the limits where either the Maxwell or the ChernSimons terms disappear; in neither case electron-electron bound states show up. However, numerical calculations indicate that fermion-fermion bound states do exist in the general case.

Motivated by recent discussions [1] about the consistency of the nonrelativistic limit of certain relativistically invariant quantum field theories, we consider in this letter the problem of determining the effective electron-electron low energy potential arising from the Maxwell-Chern-Simons (MCS) theory. We shall also investigate whether this potential defines a physically sensible and nontrivial quantum dynamics. In particular, the existence of electronelectron bound states is one of our main concerns in this work.

As known [2], the MCS theory is a (2+1)-dimensional model describing the coupling of charged fermions $(\bar{\psi}, \psi)$ of mass $m$ and electric charge $e$ to the gauge field potential $A_{\mu}$ via the lagrangian density

$$
\begin{aligned}
\mathscr{L} & =-\frac{1}{4} a F_{\mu \nu} F^{\mu \nu}+\frac{1}{4} \theta \epsilon^{\mu \nu \alpha} F_{\mu \nu} A_{\alpha} \\
& -\frac{1}{2 \lambda}\left(\partial_{\mu} A^{\mu}\right)\left(\partial_{\nu} A^{\nu}\right)+\frac{1}{2} \mathrm{i} \bar{\psi} \gamma^{\mu} \partial_{\mu} \psi \\
& -\frac{1}{2} \mathrm{i}\left(\partial_{\mu} \bar{\psi}\right) \gamma^{\mu} \psi-e \bar{\psi} \gamma^{\mu} A_{\mu} \psi-m \bar{\psi} \psi,
\end{aligned}
$$

where $F_{\mu \nu} \equiv \partial_{\mu} A_{\nu}-\partial_{\nu} A_{\mu}, \theta \geqslant 0$ is the topological mass, $\lambda$ is a gauge parameter and $a$ is a dimensionless real

* Supported in part by Conselho Nacional de Desenvolvimento Científico e Tecnológico (CNPq), Brazil. parameter $(0 \leqslant a \leqslant 1)$ enabling us to modulate the intensity of the Maxwell term. Throughout this paper we use natural units $(c=\hbar=1)$. Our metric is $g_{00}=-g_{11}=-g_{22}=1$, while for the $\gamma$ matrices we adopt the representation $\gamma^{0}=\sigma^{3}, \gamma^{1}=\mathrm{i} \sigma^{1}, \gamma^{2}=\mathrm{i} \sigma^{2} ; \sigma^{i}$, $i=1,2,3$, are the Pauli spin matrices. Neither parity nor time-reversal are, separately, symmetries of the model.

The contribution of order $e^{2}$ to the elastic scattering amplitude $\mathrm{e}^{-}+\mathrm{e}^{-} \rightarrow \mathrm{e}^{-}+\mathrm{e}^{-}$(Möller scattering) is given by

$$
\mathscr{M}_{\mathrm{fi}}=\mathscr{M}_{\mathrm{fi}}^{\mathrm{direc}}-\mathscr{M}_{\mathrm{fi}}^{\text {exchange }}
$$

where

$$
\begin{aligned}
& \mathscr{M}_{\mathrm{fi}}^{\mathrm{direct}}=\frac{-e^{2}}{2 \pi} \delta^{(3)}\left(p_{1}^{\prime}+p_{2}^{\prime}-p_{1}-p_{2}\right) \\
& \quad \times\left[\bar{v}^{(+)}\left(\boldsymbol{p}_{1}^{\prime}\right) \gamma^{\mu} v^{(-)}\left(\boldsymbol{p}_{1}\right)\right] D_{\mu \nu}(k) \\
& \quad \times\left[\bar{v}^{(+)}\left(\boldsymbol{p}_{2}^{\prime}\right) \gamma^{\nu} v^{(-)}\left(\boldsymbol{p}_{2}\right)\right] .
\end{aligned}
$$

Here, $\boldsymbol{p}_{1}, \boldsymbol{p}_{2}\left(\boldsymbol{p}_{1}^{\prime}, \boldsymbol{p}_{2}^{\prime}\right)$ are the on-shell two-momenta of the initial (final) electrons, $k \equiv p_{1}^{\prime}-p_{1}=p_{2}-p_{2}^{\prime}$ is the transferred momentum and $D_{\mu \nu}(k)$ designates the free photon propagator. One can easily check that 
$D_{\mu \nu}(k)=\frac{-\mathrm{i}}{a k^{2}-\theta^{2} / a}\left(P_{\mu \nu}-\mathrm{i} \frac{\theta}{a} \epsilon_{\mu \nu \rho} \frac{k^{\rho}}{k^{2}}\right)-\mathrm{i} \lambda \frac{k_{\mu} k_{\nu}}{k^{4}}$,

where $P_{\mu \nu} \equiv g_{\mu \nu}-k_{\mu} k_{\nu} / k^{2}$. Notice that $D_{\mu \nu}(k)=$ $D_{\nu \mu}(-k)$ and, moreover, that all terms of the form $k_{\mu} k_{\nu}$ in $D_{\mu \nu}$ do not contribute to the amplitude, since the photon propagator is contracted into conserved currents. The amplitude $\mathscr{M}_{\mathrm{fi}}^{\text {exchange }}$ follows by exchanging in $\mathscr{H}_{\tilde{\mathrm{f}}}^{\mathrm{direct}}$ the quantum numbers of the electrons in the outgoing channel.

The two-component spinor $v^{(-)}(\boldsymbol{p})\left(\bar{v}^{(+)}(\boldsymbol{p})\right)$ describes a free electron of two-momentum $p$ and energy $p^{0}=+\left(\boldsymbol{p}^{2}+m^{2}\right)^{1 / 2}$ in the initial (final) state and, therefore, obeys the free Dirac equation $\left(\gamma^{\mu} p_{\mu}-m\right) v^{(-)}(\boldsymbol{p})=0$ or, equivalently,

$H v^{(-)}(\boldsymbol{p})=p^{0} v^{(-)}(\boldsymbol{p})$,

where

$H=-\sigma^{2} p^{1}+\sigma^{1} p^{2}+m \sigma^{3}$

The solution of eq. (5) is found to be

$v^{(-)}(\boldsymbol{p})=\left(\frac{p^{0}+m}{2 p^{0}}\right)^{1 / 2}\left(\begin{array}{c}1 \\ \frac{p^{2}-\mathrm{i} p^{1}}{p^{0}+m}\end{array}\right)$.

Our normalization is $v^{(-)+}(\boldsymbol{p}) v^{(-)}(\boldsymbol{p})=1$, which in turns implies that

$$
\begin{gathered}
\bar{v}^{(+)}(\boldsymbol{p}) v^{(-)}(\boldsymbol{p})=\overline{v^{(-)}}(\boldsymbol{p}) v^{(-)}(\boldsymbol{p}) \\
=v^{(-) \dagger}(\boldsymbol{p}) \gamma^{0} v^{(-)}(\boldsymbol{p})=+\frac{m}{p^{0}} .
\end{gathered}
$$

The computation of $\mathscr{M}_{\mathrm{fi}}^{\text {direct }}$, in eq. (3), is now straightforward and yields

$$
\begin{gathered}
\mathscr{M}_{\mathrm{i} i}^{\mathrm{direct}} \simeq-\frac{\mathrm{i} e}{2 \pi} \delta^{(3)}\left(p_{1}^{\prime}+p_{2}^{\prime}-p_{1}-p_{2}\right) \\
\times\left(\frac{e}{a \boldsymbol{k}^{2}+\theta^{2} / a}-\frac{\theta}{m a} \frac{e}{a \boldsymbol{k}^{2}+\theta^{2} / a}\right. \\
\left.+\frac{2 \mathrm{i} \theta}{m a} \frac{e}{\boldsymbol{k}^{2}\left(a \boldsymbol{k}^{2}+\theta^{2} / a\right)}(\boldsymbol{k} \times \boldsymbol{p})\right)
\end{gathered}
$$

where only zero- and first-order terms in $|\boldsymbol{p}| / m$ have been retained. Furthermore, we designated by $\boldsymbol{p} \equiv \frac{1}{2}\left(\boldsymbol{p}_{1}-\boldsymbol{p}_{2}\right)$, the relative linear momentum of the incoming electrons in the center of mass frame of reference.

The first term in the large parentheses of $(8)$ is the expected repulsive two-dimensional Yukawa potential, slightly modified by the presence of the parameter $a$. Its Fourier transform is found to read

$$
\begin{aligned}
& V_{\mathrm{Y}}(a, \boldsymbol{r}) \equiv \frac{e}{(2 \pi)^{2}} \int \mathrm{d}^{2} k \frac{\mathrm{e}^{\mathrm{i} \boldsymbol{k} \cdot \boldsymbol{r}}}{a \boldsymbol{k}^{2}+\theta^{2} / a} \\
& =\frac{e}{2 \pi a} K_{0}\left(\frac{\theta r}{a}\right),
\end{aligned}
$$

where $K_{0}$ is the modified Bessel function, $\boldsymbol{r}$ is the relative distance between electrons and $r \equiv|\boldsymbol{r}|$. Of course, $V_{Y}$ diverges logarithmically as the topological mass approaches zero ( the Coulomb limit). The second term in the large parentheses of (8) describes an attractive Yukawa potential which disappears in the limit of vanishing topological mass. For $\theta>m a$ $(\theta<m a)$ the combined action of these two potentials is seen to be attractive (repulsive), whereas for $\theta=m a$ their action mutually cancels. After computing the Fourier transform of the third term in the large parentheses of $(8)$ one arrives at

$V_{L}(a, r)=-\frac{e}{\pi m \theta} \frac{1}{r^{2}}\left[1-\frac{\theta r}{a} K_{1}\left(\frac{\theta r}{a}\right)\right] L$.

The presence of the orbital angular momentum $L \equiv \boldsymbol{r} \times \boldsymbol{p}$ in the right-hand side of this last equation should be noticed. It implies that $V_{L}$ is odd under parity and time-reversal transformations. Therefore, space- and time-inversion do not leave the total potential,

$V_{\mathrm{T}}(a, \boldsymbol{r}) \equiv\left(1-\frac{\theta}{m a}\right) V_{\mathrm{Y}}(a, \boldsymbol{r})+V_{L}(a, \boldsymbol{r})$,

invariant.

We address next to the problem of quantizing the two-dimensional nonrelativistic motion of two distinguishable particles, of equal mass $m$ (reduced mass $\frac{1}{2} m$ ), interacting through the potential $V_{\mathrm{T}}$. Afterwards, it will be properly taken into account that the particles one is dealing with are indistinguishable fermions.

In polar coordinates $r, \phi$, the position representation of the operator $L$ is known to be $-\mathrm{i} \partial / \partial \phi$; the normalized eigenfunctions and eigenvalues of $L$ being, respectively, $\sqrt{(1 / 2 \pi)} \mathrm{e}^{\mathrm{i} / \phi}, l=0, \pm 1, \pm 2, \ldots$. 
The hamiltonian describing the relative quantum dynamics of the two-body system, then, reads

$\mathscr{H}=\mathscr{H}_{0}+e V_{\mathrm{T}}(a, \boldsymbol{r})$,

where

$\mathscr{H}_{0}=-\frac{1}{m}\left(\frac{\partial^{2}}{\partial r^{2}}+\frac{1}{r} \frac{\partial}{\partial r}\right)-\frac{1}{m r^{2}} \frac{\partial^{2}}{\partial \phi^{2}}$

is the free hamiltonian. The bound states of $\mathscr{H}$, referred to as $\Psi_{n l}(r, \phi)$, will be common eigenstates of $\mathscr{H}$ and $L$, i.e., $\Psi_{n l}(r, \phi)=R_{n l}(r) \mathrm{e}^{l \phi}$. Here, $R_{n l}(r)$ is the $n$th normalizable eigenfunction of the radial hamiltonian $\mathscr{H}_{l}$ and we shall call $E_{n t}$ the corresponding eigenvalue. Hence,

$$
\begin{aligned}
& \mathscr{H}_{l} R_{n l}=-\frac{1}{m}\left(\frac{\partial^{2} R_{n l}}{\partial r^{2}}+\frac{1}{r} \frac{\partial R_{n l}}{\partial r}\right)+U_{l}^{\text {eff }} R_{n l} \\
& =E_{n l} R_{n l},
\end{aligned}
$$

where $U_{l}^{\text {eff }}$,

$$
\begin{aligned}
& U_{l}^{\mathrm{eff}}(a, r)=\frac{l^{2}}{m r^{2}}+e V f^{(l)}(a, r) \\
& =\frac{l^{2}}{m r^{2}}+\frac{e^{2}}{2 \pi a}\left(1-\frac{\theta}{m a}\right) K_{0}\left(\frac{\theta r}{a}\right) \\
& -\frac{e^{2}}{\pi \theta} \frac{l}{m r^{2}}\left[1-\frac{\theta r}{a} K_{1}\left(\frac{\theta r}{a}\right)\right],
\end{aligned}
$$

denotes the $l$ th partial wave effective potential. Of course, $V_{\mathrm{T}}^{(l)}(a, r)$ has been read off directly from (10) and (11) after the replacement $L \rightarrow l$. As for the scattering states, we start by recalling that a plane wave of momentum $\boldsymbol{k}$, pointing along the direction $\phi=0$, can be decomposed into circular waves as follows: $\mathrm{e}^{\mathrm{i} r \cdot k}=\sum_{l=-\infty}^{+\infty} \mathrm{i}^{l} J_{l}(k r) \mathrm{e}^{\mathrm{i} / \phi}$. Here, $k \equiv|\boldsymbol{k}|$ and $J_{l}$ denotes the Bessel function of integer order. Similarly, the outgoing scattering state defined by $\mathscr{H} \Psi_{k}^{(+)}(\boldsymbol{r})=E \Psi_{k}^{(+)}(\boldsymbol{r})$, with $E=k^{2} / m$, admits the partial wave decomposition

$$
\Psi_{k}^{(+)}(\boldsymbol{r})=\frac{1}{2 \pi} \sum_{l=-\infty}^{+\infty} \mathrm{i}^{l} \Omega_{l}(k ; r) \mathrm{e}^{\mathrm{i} / \phi},
$$

where $\Omega_{I}(k ; r)$ verifies the Lippmann-Schwinger radial integral equation

$$
\begin{aligned}
& \Omega_{l}(k ; r)=J_{l}(k r) \\
& +2 \pi \int_{0}^{\infty} \mathrm{d} r^{\prime} r^{\prime} G_{k}^{(l)}\left(r, r^{\prime}\right) e V_{+}^{(l)}\left(a, r^{\prime}\right) \Omega_{l}\left(k ; r^{\prime}\right)
\end{aligned}
$$

and $G_{k}^{(l)}\left(r, r^{\prime}\right)$ designates the $l$ th component of the resolvent $\left\langle\boldsymbol{r}\left|\left(E+\mathrm{i} \epsilon-\mathscr{H}_{0}\right)^{-1}\right| \boldsymbol{r}^{\prime}\right\rangle$. A simple calculation shows that

$G_{k}^{(l)}\left(r, r^{\prime}\right)=-\frac{1}{4} \mathrm{im} J_{l}\left(k r_{<}\right) H\left(^{\prime \prime}\left(k r_{>}\right)\right.$,

where $r_{<}\left(r_{>}\right)$is the smaller (the larger) of $r$ and $r^{\prime}$ and $H\}^{1)}$ is the Hankel function of integer order.

Before embarking into the problem of solving (12) and/or (15) for the general case, represented by the potential (11), we analyze some limiting situations which are tractable by analytic means. As seen from (1), for $a=1, \theta=0$ the MCS theory reduces to $\mathrm{QED}_{3}$ and, correspondingly, the potential $V_{\mathrm{T}}$ collapses into the repulsive Coulomb potential, $V_{\mathrm{C}}=-(e / 2 \pi)$ $\times \ln \left(r / r_{0}\right)$, where $r_{0}$ is an infrared regulator. The logarithmic potential in two space dimensions has been widely studied in the past [3-5]. In the repulsive case the energy spectrum is continuous, while bound states only show up in the attractive case. The interesting thing in connection with the Coulomb potential in two dimensions is that the s-wave hamiltonian $\mathscr{H}_{l=0}$ only becomes essentially self-adjoint, on its natural domain of functions, after an appropriate extension [3].

We are chiefly interested in the limit $a=0, \theta \neq 0$, where the Maxwell term disappears and the MCS theory degenerates into the Chern-Simons (CS) theory. Formal developments indicate that, in this case, composite fields obeying fractional statistics can be constructed [6-8]. One can readily check that the potential $V_{\mathrm{t}}^{(\prime)}$ now reduces to

$$
V_{\dagger}^{(l)}(a=0, r)=-\frac{e}{\pi \theta m}\left(\frac{1}{2 r} \delta(r)+\frac{1}{r^{2}} l\right) .
$$

For arriving at this last equation we have considered as equivalents the distributions $\delta^{2}(r)$ and $(1 / 2 \pi r)$ $\times \delta(r)$, which is strictly true if one is restricted to work with single-valued functions. The delta potential in (16) only acts on the s-wave. Indeed, by replacing (16) into (15) one finds, for the s-wave, the algebraic relation 
$\Omega_{0}(k ; r)=J_{0}(k r)+\frac{\mathrm{i} e^{2}}{4 \theta} H_{0}^{(1)}(k r) \Omega_{0}(k ; 0)$,

whereas the $l \neq 0$ partial waves are required to verify the integral equation

$$
\begin{aligned}
& \Omega_{l}(k ; r) \\
& \left.=J_{l}(k r)+\frac{\mathrm{i} e^{2}}{2 \theta} l H\right\rangle^{(1)}(k r) \int_{0}^{r} \frac{\mathrm{d} r^{\prime}}{r^{\prime}} J_{l}\left(k r^{\prime}\right) \Omega_{l}\left(k ; r^{\prime}\right) \\
& \left.+\frac{\mathrm{i} e^{2}}{2 \theta} l J_{l}(k r) \int_{r}^{\infty} \frac{\mathrm{d} r^{\prime}}{r^{\prime}} H\right\rangle^{(1)}\left(k r^{\prime}\right) \Omega_{l}\left(k ; r^{\prime}\right) .
\end{aligned}
$$

As usual, the expression giving the scattering amplitude $f(k, \phi)$ in terms of the phase shifts $\left(\delta_{l}(k)\right)$,

$f(k, \phi)=-\frac{\mathrm{i}}{\sqrt{2 \pi k}} \sum_{l=-\infty}^{+\infty}\left(\mathrm{e}^{2 \mathrm{i} \delta l(k)}-1\right) \mathrm{e}^{\mathrm{i} / \phi}$,

is derived by studying the asymptotic behavior $(r \rightarrow \infty)$ of $\Psi_{k}^{(+)}(\boldsymbol{r})$. Presently,

$\mathrm{e}^{2 \mathrm{i} \delta \delta_{0}(k)}=1+\frac{\mathrm{i} e^{2}}{2 \theta} \Omega_{0}(k ; 0)$

and

$\mathrm{e}^{2 \mathrm{i} \delta_{l}(k)}=1+\frac{\mathrm{i} e^{2} l}{\theta} \int_{0}^{\infty} \frac{\mathrm{d} r}{r} J_{l}(k r) \Omega_{l}(k ; r)$,

if $l \neq 0$. From $(18)$ one obtains $\Omega_{l}(k ; 0)=0$ for all $l \neq 0$, as required by the single-valuedness of $\Psi_{k}^{(+)}(r)$ (see eq. (14)). On the other hand, for $e^{2}$ a finite and nonvanishing positive constant, eq. (17) turns out to be inconsistent. In fact, after controlling the logarithmic singularity in $H_{0}^{(1)}$ by means of the ultraviolet cutoff $A$ one finds [1]

$\Omega_{0}(k ; 0)=\left\{1-\frac{e^{2}}{4 \pi \theta}\left[\mathrm{i} \pi+2 \ln \left(\frac{A}{k}\right)\right]\right\}^{-1}$.

Obviously, $\Omega_{0}(k ; 0) \rightarrow 0$ as $A \rightarrow \infty$. When this result is fed back into (17) one obtains, in view of $J_{l}(0)=1$, $\Omega_{0}(k ; 0)=1 \neq 0$, which is contradictory. As pointed out in ref. [1], the lack of self-adjointness of $\mathscr{H}_{l=0}$ is at the root of this difficulty. The self-adjoint extension of $\mathscr{H}_{l=0}$, required to render the theory consistent, has been shown [1] to be equivalent to the introduction of the renormalized coupling constant $g^{2}$,
$\frac{1}{g^{2}(M)} \equiv \frac{1}{e^{2}(A)}-\frac{1}{2 \pi \theta} \ln \left(\frac{A}{M}\right)$

where $M$ is a subtraction point and the bare coupling constant $e^{2}$ is taken to be cutoff dependent. Instead of (22) one can, then, write

$e^{2} \Omega_{0}(k ; 0)=\left[\frac{1}{g^{2}(M)}-\frac{1}{2 \pi \theta} \ln \left(\frac{M}{k}\right)-\frac{\mathrm{i}}{4 \theta}\right]^{-1}$.

In words, as $A \rightarrow \infty, e^{2}(\Lambda) \rightarrow 0$ and $\Omega_{0}(k ; 0 ; A) \rightarrow \infty$ in such a way that the product $e^{2} \Omega_{0}(k ; 0)$ remains finite. The immediate consequence is that only s-wave scattering takes place, as seen from (17) and (18). From (17) and (24) also follows that $\Omega_{0}(k ; r)$ has a pole located on the positive imaginary axis of the $k$ complex plane. Therefore, this pole signalizes for a bound state whose energy can be corroborated to be

$E_{\mathrm{B}}=-\frac{M^{2}}{m} \exp \left(-\frac{4 \pi \theta}{g^{2}}\right)$.

The corresponding bound state eigenfunction is found by computing the residue of $\Omega_{0}(k ; r)$ at the pole and reads

$\Psi_{\mathrm{B}}=\sqrt{-2 m E_{\mathrm{B}}} K_{0}\left(\sqrt{-m E_{\mathrm{B}}} r\right)$.

Hence, $\mathscr{H}_{l=0}$ has been extended so as to remain selfadjoint with respect to square integrable functions that are not finite at the origin. We emphasize that the attractive character of the delta potential in (16) bears no relation whatsoever with the sign of the coupling constant $e^{2}$.

We now recall that the particles we are dealing with are fermions. The scattering amplitude for indistinguishable particles verifying Fermi-Dirac statistics $(f(k, \phi))$ is constructed from $f(k, \phi)$ as follows: $\bar{f}(k, \phi)=f(k, \phi)-f(k, \phi+\pi)$. After taking into account eq. (19) one, then, arrives to

$\bar{f}(k, \phi)=-\frac{2 \mathrm{i}}{\sqrt{2 \pi k}} \sum_{\text {odd }}\left(\mathrm{e}^{2 \mathrm{i} \delta(k)}-1\right) \mathrm{e}^{\mathrm{i} l \phi}$.

We, therefore, conclude that, within the present formulation of the quantum dynamics, the electrons remain free.

We shall next investigate whether an alternative formulation of the problem might lead to solutions showing the existence of an effective electron-elec- 
tron interaction. As before, we start by regulating the troublesome s-wave in order for $f(k, \phi)-f(k, \phi+\pi)$ to be a mathematically sound operation. After arriving at (25) the regulator is removed, but this time the coupling constant is not renormalized. Then, all waves contributing to $\bar{f}(k, \phi)$ do interact. From eqs. (13) and (16), and since only the s-wave is affected by the delta interaction, follows that the $l$ th partial wave effective potential is now given by

$$
U_{l}^{\mathrm{eff}}(a=0, r)=\frac{1}{m r^{2}} \nu^{2}(l), \quad l= \pm 1, \pm 3, \ldots,
$$

where $\nu^{2}(l) \equiv l^{2}-\alpha l$ and $\alpha \equiv e^{2} / \theta \pi$. We, then, focus on solving the radial Schrödinger equation

$$
\frac{1}{r} \frac{\mathrm{d}}{\mathrm{d} r}\left(r \frac{\mathrm{d} \Omega_{l}(k ; r)}{\mathrm{d} r}\right)+\left(k^{2}-\frac{\nu^{2}(l)}{r^{2}}\right) \Omega_{l}(k ; r)=0 .
$$

As we already mentioned, for $l \neq 0$ single-valuedness requires $\Omega_{l}(k ; 0)=0$. On the other hand, bound states are to be described by normalizable wave functions, namely, $\int_{0}^{\infty} \mathrm{d} r r\left|\Omega_{l}(k ; r)\right|^{2}<\infty$, while scattering states must verify the asymptotic condition

$$
\begin{aligned}
& \lim _{r \rightarrow \infty} \Omega_{l}(k ; r) \rightarrow \sqrt{\frac{2}{\pi k r}} \mathrm{e}^{\mathrm{i} \delta_{l}(k)} \\
& \quad \times \cos \left[k r-\frac{1}{2} \pi l-\frac{1}{4} \pi+\delta_{l}(k)\right],
\end{aligned}
$$

as seen from eq. (18). For negative values of $l$, $\nu^{2}(l)>0$, both terms contributing to $U_{l}^{\text {eff }}$ are repulsive and, therefore, only scattering states will be observed. The solution to eq. (27), verifying the boundary conditions, is

$\Omega_{l}(k ; r)=\mathrm{e}^{\mathrm{i} \delta_{l}(k)} J_{\nu(l)}(k r)$.

By comparing eqs. (28) and (29) one easily finds that

$\delta_{l}=\frac{1}{2} \pi|l|\left(1-\sqrt{1+\frac{\alpha}{|l|}}\right)<0$.

The lack of dependence of $\delta_{l}$ on $k$ is not a surprise, since (27) is an almost free Schrödinger equation. Furthermore, notice that for $|l| \gg \alpha, \delta_{l}(k)=-\frac{1}{4} \pi \alpha$ $=-e^{2} / 4 \pi=$ constant, implying that the phases of all these partial waves are shifted by the same amount. For positive values of $l$, and such that $\nu^{2}(l)>0$, the effective potential is still repulsive, although that part of $V_{\mathrm{f}}^{(l)}(a=0, r)$ contributing to $U_{l}^{\text {eff }}$ is now attractive (see eqs. (13) and (16)). Hence, the solution to eq. (27) is again given by (29), but for the phase shifts one obtains

$\delta_{l}=\frac{1}{2} \pi l\left(1-\sqrt{1-\frac{\alpha}{l}}\right)>0$.

Finally, for $l>0$ but with $\nu^{2}<0$ the effective potential becomes attractive and we can entertain the possibility of an electron-electron bound state. To investigate the existence of bound states, we replace in (27) $k=\mathrm{i} \kappa$ and $\nu(l)=\mathrm{i} p(l)$. The only normalizable solution to the differential equation thus obtained is $H_{\mathrm{i} p}^{(1)}(\mathrm{i} \kappa r)$, since

$H_{i p}^{(1)}(\mathrm{i} \kappa r) \rightarrow \sqrt{\frac{2}{\mathrm{i} \pi \kappa r}} \mathrm{e}^{-\kappa r+p \pi / 2-\mathrm{i} \pi / 4}$

as $r \rightarrow \infty$. However, at the origin,

$\lim _{r \rightarrow 0} H_{i p}^{(1)}(\mathrm{i} \kappa r) \rightarrow 2 \mathrm{i}\left(\frac{\mathrm{e}^{p \pi / 2}}{\sinh \pi p}\right) \sin \left[p \ln \left(\frac{1}{2} \kappa r\right)\right]$,

the function does not vanish but rather oscillates with ever increasing frequency as $r \rightarrow 0$. The behavior of $H_{\mathrm{i} p}^{(1)}(\mathrm{i} \kappa r)$ at the origin shows that, as it happens in three space dimensions [9], the attractive $1 / r^{2}$ potential does not define a consistent quantum mechanical problem. This inconsistency is also present in the scattering solutions which are found to read

$\Omega_{l}(k ; r)=\frac{1}{2} \mathrm{e}^{\mathrm{i} \delta l}\left[H_{\mathrm{i} p}^{(1)}(k r)+H_{\mathrm{i} p}^{(2)}(k r)\right]$,

with

$\delta_{l}=[\mathrm{i} p(l)+l] \cdot \frac{1}{2} \pi$,

violating the reality of the phase shifts. The consistency of the whole approach demands, then, the elimination of this sector, which can be done by restricting $\alpha$ to be less than 1 or, what amounts to the same thing, $\theta>e^{2} / \pi$. To summarize: there exists an alternative formulation of the quantum dynamics generated by the CS potential according to which the electrons interact. However, no bound state of the two electron system exists.

We return now to the general problem defined by (12) and (13). In terms of $\tilde{\theta} \equiv \theta / a$ and of the dimensionless quantities $y \equiv \theta r / a, \alpha, \beta \equiv m / \bar{\theta}$ and $\epsilon_{n l} \equiv$ $m E_{n l} / \widetilde{\theta}^{2}$, eq. (12) can be casted as follows: 
$\left(\frac{\partial^{2} R_{n l}}{\partial y^{2}}+\frac{1}{y} \frac{\partial R_{n l}}{\partial y}\right)-\tilde{U}_{l}^{\mathrm{eff}} R_{n l}+\epsilon_{n l}=0$,

where

$$
\begin{gathered}
\tilde{U}_{l}^{\mathrm{eff}}(a, y)=\frac{l^{2}}{y^{2}}-\frac{1}{2} \alpha(1-\beta) K_{0}(y) \\
-\frac{\alpha l}{y^{2}}\left[1-y K_{1}(y)\right] .
\end{gathered}
$$

A rough analysis based on the uncertainty principle leads to the condition

$$
(\Delta y)^{2}\left|\tilde{U}_{l}^{\text {eff }}\right| \geqslant 1
$$

for the existence of bound states. A numerical study of the potential $\tilde{U}_{l}^{\text {eff }}$ shows that this condition is verified for several values of $\alpha$ and $\beta$. In particular, it can be seen to hold for some values of $\alpha$ and $\beta$ within the region $\alpha / \beta \ll 1$, where the field theoretical perturbative approximation is justified. We are gratefully indebted to Professor J. Lyra for helping us in numerically solving eq. (34) for $l=1, a=1, \alpha=500$ and $\beta=10^{5}$. The ground state eigenvalue was found to be $\epsilon_{01}=-1.19 \pm 10^{-3}$. A thorough study of the eigenvalue structure, as well as of the modifications of the effective potential induced by radiative corrections, will be presented elsewhere.

We then conclude that the Chern-Simons term alone is unable to form "Cooper pairs". The presence of the Maxwell term provides an stabilizing mechanism allowing for the existence of "Cooper pairs".

\section{References}

[1] R. Jackiw, MIT preprint CTP \#1937(1991).

[2] S. Deser, R. Jackiw and S. Templeton, Ann. Phys. (NY) 140 (1982) 372.

[3] F. Gesztesy and L. Pittner, J. Phys. A 11 (1978) 679.

[4] F.J. Asturias and R. Aragon, Am. J. Phys. 53 (1985) 893.

[5] K. Eveker, D. Grow, B. Jost, C.E. Montfort III, K.W. Nelson, C. Stroh and R.C. Witt, Am. J. Phys. 58 (1990) 1183.

[6] G.W. Semenoff, Phys. Rev. Lett. 61 (1988) 517.

[7] A. Foerster and H.O. Girotti, Phys. Lett. B 230 (1989) 83; Nucl. Phys. B 342 (1990) 680.

[8] A. Foerster and H.O. Girotti, Statistical transmutation in $2+1$ dimensions, in: J.J. Giambiagi Festschrift, eds. H. Falomir, R.E. Gamboa Saravi, P. Leal Ferreira and F.A. Schaposnik (World Scientific, Singapore, 1990) p. 161.

[9] P.M. Morse and H. Feshbach, Methods of theoretical physics (McGraw-Hill, New York, 1953) p. 1667. 